\title{
Cardiovascular Pre-participation Screening for Young Competitive Athletes
}

\author{
Denise Law-Vinh ${ }^{1}$, Wyanne Law, $\mathrm{BScH}^{2}$ \\ ${ }^{1}$ Faculty of Science, University of Ottawa \\ ${ }^{2}$ Faculty of Medicine, University of Ottawa
}

\section{A BSTRACT}

This commentary discusses pre-participation cardiovascular screening for young competitive athletes. Canada currently does not have a consolidated document of guidelines for such screening procedure, even though athletes with pre-existing cardiovascular conditions may experience sudden cardiac death (SCD) during intensive training or competitions. How can we balance the safety of athletes while considering the costs of implementing such program and the downstream effects of athletes who may be barred from pursuing their dream sport upon diagnosis? This commentary examines the common causes of SCD, components of the screening procedure, costbenefit analyses of pre-participation screening, and management strategies upon diagnosis.

\section{RÉ S U É}

Ce commentaire aborde le dépistage cardiovasculaire chez les jeunes athlètes de compétition avant la participation. Le Canada ne dispose actuellement pas de lignes directrices relativement à ce type de dépistage, même si un athlète qui aurait une affection préexistante pourrait être victime de mort cardiaque subite lors d'un entraînement intensif ou d'une compétition. Comment pouvons-nous équilibrer la sécurité des athlètes et tenir compte du coût pour mettre un tel programme sur pied, puis gérer les effets en aval pour des athlètes qui pourraient être interdits de poursuivre leur rêve sur la réception d'un diagnostic? Ce commentaire aborde les causes courantes de la mort cardiaque subite, les composantes du processus de dépistage, une analyse coût/bénéfice du dépistage avant la participation, de même que des stratégies pour gérer les effets d'un diagnostic.

\section{INTRODUCTION}

Recently in the news there have been several cases of young competitive athletes who have collapsed in the middle of their game due to pre-existing cardiac problems. Rich Peverley, a 31-year-old hockey player, collapsed on the Columbus Blue Jackets-Dallas Stars' bench in the middle of the game due to an underlying arrhythmia on March 11th, 2014 [1]. Collapses that lead to sudden death have been vividly captured by the media and have raised concerns in the medical community, as they serve to remind us that even young, healthy athletes can succumb to cardiovascular abnormalities. Each year in Canada, unsuspected sudden cardiac events cause approximately 500 deaths in people under the age of 40 in the general population [1]. For the purposes of this article, competitive young athletes are defined as those in high school or those pursuing post-secondary education, between the ages of 14-22, and participate in any organized or individual sport that demands systematic training and regular competition, and rewards athletic excellence [3]. Sudden cardiac events are the leading cause of non-traumatic sudden death in athletes worldwide [2]. To prevent such devastating deaths at the

Keywords: sudden cardiac death, athletes, cardiovascular screening prime of a young competitive athletes' career, pre-participation cardiovascular screening is a systematic way to identify those who have pre-existing conditions that may lead to sudden death. The American Heart Association (AHA), European Society of Cardiology (ESC), and International Olympic Committee (IOC) have set their own recommendations regarding pre-participation cardiovascular screening $[2,3,4]$. Most of these guidelines are based on a 25 -year-long Italian observational study, as no clinical trials have been done to date [5]. A consensus document of guidelines has yet to be set for young competitive Canadian athletes participating in high school or post-secondary-level varsity sports. For example, at the University of Ottawa, a "certified team doctor annually screens all players during [their] 1-2 week training camp in August before school begins," and there is "more in depth screen for rookie players" [6]. This article will explore the cost-benefit analysis of such screening procedure, as well as management strategies for athletes who have positive results.

\section{CAUSES OF SUDDEN DEATH IN ATHLETES}

There are 1101 reported cases of sudden cardiac deaths athletes under the age of 35 from years 1966 to 2004 [1]. Soccer, basketball and running are sports that are most frequently 
involved in sudden cardiac death (SCD) [2]. Approximately half of these athletes had congenital anatomical heart disease and cardiomyopathies, and $10 \%$ had early-onset atherosclerotic coronary disease [2].

Common congenital anomalies for SCD are anomalous origin of the coronary artery and hypertrophic cardiomyopathy (HCM) [5]. Anomalous origin of the coronary artery is often associated with structural forms of congenital heart disease. HCM occurs when a portion of the myocardium and the septum is thickened, causing dynamic outflow obstruction for blood to enter to systemic circulation. Arrhythmogenic right ventricular cardiomyopathy (ARVC) is the most common type of cardiomyopathy in SCD patients [5]. This occurs when the right ventricle and the subepicardial region of the left ventricle are replaced by fibrofatty tissue, thus increasing the risk of life-threatening tachyarrhythmias. Atherosclerotic coronary disease can lead to death when plaque on the vessel wall breaks off and occludes an important coronary artery that can result in myocardial infarction [5]. Under strenuous exercise, or situations of dehydration or vasodilation that decreases preload to the heart, these pre-existing conditions can cause SCD in asymptomatic athletes.

\section{COST-BENEFIT ANALYSES OF PRE-PARTICIPATION SCREENING}

The rationale of cardiovascular pre-participation screening is to identify young competitive athletes who may be at risk of sudden cardiac death. Whether screening is economically justifiable from a public health standpoint is debatable. The incidence of sudden cardiac death among young competitive athletes is about 1 in 50,000 athletes, which is much lower than that of the general population (1 in 300) [8]. Furthermore, there are many different components of a screening protocol that can be very costly. AHA, ESC and IOC all advocate for a cardiovascular history and physical exam for young athletes in college and professional sports (Figure 1), which saves 0.56 life-year per 1000 athletes compared to no screening [9].These first steps can identify cardiomyopathy and other genetic cardiac conditions. Furthermore, AHA and IOC recommend the screening to be supplemented by a 12-lead rest electrocardiogram (ECG) to identify rhythm, conduction and repolarization abnormalities $[2,3]$. For those between the ages of 18 to 29, ECG screening has a low sensitivity of $46 \%$ and an excellent specificity of $100 \%$ for familial HCM [11]. Although ECG screening is not very sensitive and may capture some false positives for cardiovascular diseases, it is an effective first step that can save money from conducting an unnecessary workup. Adding an additional ECG test to screen for abnormalities saves 2.06 life years per 1000 athletes at a cost of $\$ 42,000$ per life-year saved compared to cardiovascular history and physical exam alone after sensitivity analyses [9]. The intervention of ECG would be considered cost-effective, as it is less than the traditional cost-benefit benchmark of $\$ 50,000$ per life-year gained (approximately the cost of dialysis per year) [10].

IOC also mandates that if cardiovascular history, physical exam and ECG findings are positive, then athletes should be referred to an age-appropriate specialist [2]. The cardiac workup done by the specialist may include an echocardiogram, stress test, 24-hour Holter monitoring, cardiac MRI (CMR), cardiac CT, radionuclide angiogram and electrophysiology studies. These additional investigations are necessary for diagnosis of certain cardiovascular conditions [8]. For example, the definitive diagnosis of HCM is done by echocardiography. However, the diagnostic value of an echocardiogram and ECG for familial HCM is not significantly different from ECG alone. For those between the ages of 18 and 29 , the sensitivity only increases by $4 \%$ with further screening using echocardiogram, and specificity remains at $100 \%$ [11]. If echocardiac images are suboptimal, CMR is recommended for those who have a high index of suspicion for $\mathrm{HCM}$, or alternatively cardiac CT for those who have implantable cardioverter defibrillator (ICD) or pacemakers [12]. Other cardiovascular diseases may also require further testing. The diagnoses of other diseases may also require modalities beyond ECG screening [12]. Coronary artery disease can be ruled out by coronary angiography. Acute aortic dissection can be excluded by transesophageal echocardiography. Genetic tests are newer, more definitive measures of hereditary cardiac conditions. These tests, if added as part of mandatory pre-participation screening for young competitive athletes, can place a stress on Medicare in Canada.

Although the IOC has suggested further workup for young competitive athletes who have positive cardiovascular history, physical exam and ECG findings, Medicare in Canada may not have the necessary infrastructure to support this protocol. In Ontario alone, 498 million Canadian dollars have been spent on echocardiograms from 1992 to 2001 [13]. There is an increasing usage of cardiac technologies over this 10 -year span, and a total of $\$ 2.87$ billion expenditure would need to be invested to meet the cost-benefit benchmark of $\$ 50,000$ per life-year gained. Although only a handful of athletes would have positive findings that lead to extensive cardiac workup, further cost-benefit analyses should be conducted before implementing mandatory preparticipation screening. These tests, nonetheless, ensure that athletes with underlying cardiovascular conditions are training safely.

\section{MANAGEMENT STRATEGIES AFTER DIAGNOSIS}

Despite the benefits of pre-participation cardiovascular screening before admission to a varsity team, it may bring about psychological turmoil to young competitive athlete. There are various management strategies to cope with the diagnosis, such as psychotherapy, maintaining a positive outlook, lifestyle changes and pharmacological interventions. Athletes who are identified at risk are not alone; family, relatives, coaches and teammates are affected as well. Although sudden cardiac death (SCD) does occur, it is not a topic that is discussed casually among friends. In a news article, a father wrote about his feelings towards his son's death from a heat stroke in the middle of a football game in Arkansas. The father had wished that his 


\section{Commentary}

son's death would promote the importance of pre-screening that could prevent heart diseases from occurring. Others around who witnessed his son's death had treated this event as a, "freak thing," which is something abnormal and rare [19].

\section{Psychotherapy}

Emotional stress plays a factor on athletes who have pre-existing cardiovascular conditions [14]. For example, emotional stress can lead to hypertrophic cardiomyopathies [15]. In addition, athletes who experience anger may encounter spontaneous ventricular and atrial arrhythmias leading to SCD [15]. These athletes may suffer from emotional stress because they are young and otherwise appear healthy, and results from the pre-participation screening can have a negative effect on their dreams of becoming a professional athlete and discourage them from further intense training.

Psychotherapy helps to ease the mental stress for susceptible individuals. Patients with $\mathrm{HCM}$ attending specialty clinics demonstrate lower levels of anxiety and depression [14]. Support groups and clinics can also positively influence the mentality of individuals who feel vulnerable. Test results indicating an underlying cardiovascular condition can eliminate a patient's doubt and motivate positive behavioural changes [20]. He or she is now aware to refrain themselves from participating in intense sports. On the other hand, since most SCD in athletes are caused by hypertrophic cardiomyopathies (HCM) that is characterized as a genetic condition, individuals who have this condition place an additional burden on family members to get tested as well. A range of emotion such as fear, guilt, anger, denial, grief and despair can be experienced by family relatives [20].

\section{Lifestyle Modifications}

Although young competitive athletes at high risk of cardiovascular disease are encouraged to participate in fewer, less intensive sports, completely withdrawing from physical exercise or a sport may make the situation worse. Those who do so may still experience SCD or other cardiovascular disorders [14], such as changes in rhythm or electrical conduction [18]. Assuring that there will be no abnormalities in rhythm and electrical conduction of the heart, the individual should not feel any chronic pain while engaging in moderate level exercising.

In general, it is common for athletes to follow a strict dietary plan or even consume dietary supplements. However, those with cardiovascular conditions may need to modify their diets after diagnosis. For example, they may need to avoid foods like grapefruit juice that contain flavonoids, which affect the electrical depolarization and repolarization of the ventricles (QT) interval [18].

\section{Maintaining a Positive Outlook}

Young competitive athletes who are diagnosed with cardiovascular diseases and at high risk of SCD are prevented from competing in a sport they are passionate about. However, they can still be involved even if they are not recommended to participate in high intense training. Other alternatives involve: coaching, managing the team and training other athletes. These are potential positive outlooks for an individual who is put in such a situation. Regardless if one is affected from cardiovascular disease or not, if we consider coaches and professional trainers as athletes who once competed but had stopped for a certain reason, staying involved and becoming a coach/trainer is one way they keep in touch with the sport that they are passionate about. Coaching help athletes grow and become a part of others' success in winning or accomplishing their goals. Being involved as a coach can help individuals have a positive reflection on themselves.

\section{Pharmacological Interventions}

Once an individual has been identified at high risk of SCD, he or she may need to take medications to prevent SCD. For example, calcium channel blockers or beta-blockers are commonly administered to these individuals $[15,17]$. Many athletes

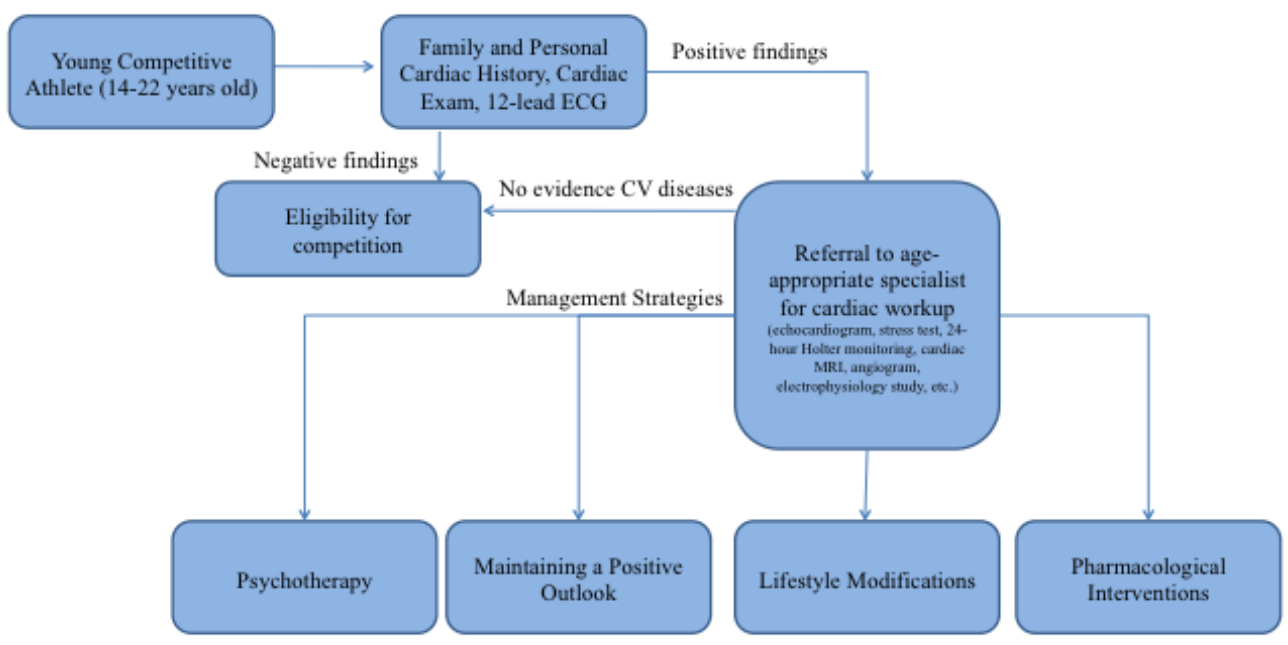

Figure 1: Steps in cardiovascular pre-participation screening and subsequent management strategies. 


\section{Commentary}

also use non-steroidal anti-inflammatory drugs (NSAIDs) to treat sports injuries, but there is a lack of knowledge on the cardiac electrophysiological effects of these compounds. NSAIDs can block certain potassium channels; consideration of risks must be taken into account when administering drugs and dietary supplements that may prolong the repolarization of the heart.

\section{CONCLUSION}

We believe that some aspects of the cardiovascular pre-participation screening should be conducted in young competitive athletes (between the ages of 14-22 in high school or post-secondary education), so that those with pre-existing cardiovascular conditions can be identified. However, it may not be economically justifiable from a public health standpoint given the low incidence of sudden cardiac death in athletes compared to the general population, marginal diagnostic value of further testing and the consideration that Canada may not have the infrastructure to support such policy. Upon finding the results of preparticipation screening, there are many management strategies to address individuals' emotional stress and risk of developing future cardiovascular events, such as psychotherapy, maintaining a positive outlook and pharmacological interventions. Further investigation should be conducted on the costs of implementing these management strategies.

\section{REFERENCES}

1. Weeks, Carly. "Benefits of cardiac screening examined after NHL player collapses." The Globe and Mail [Ottawa] 11 Mar. 2014, sec. Health: Online. Print.

2. Bille K, Figueiras D, Schamasch P, et al. "Sudden cardiac death in athletes: the Lausanne Recommendations." European Journal of Cardiovascular Prevention \& Rehabilitation 13.6 (2006): 859-875. Print.

3. Barry, Maron, Thompson P, et al. "Recommendations and Considerations Related to Preparticipation screening for cardiovascular abnormalities in competitive athletes: 2007 Update: A scientific statement from the American Heart Association Council and Metabolism: Endorsed by the American Col." Circulation 115.12 (2007): 1643-455. Print.

4. Corrado, D. "Cardiovascular Preparticipation Screening Of Young Competitive Athletes For Prevention Of Sudden Death: Proposal For A Common European Protocol: Consensus Statement Of The Study Group Of Sport Cardiology Of The Working Group Of Cardiac Rehabilitation And." European Heart Journal 26.5 (2004): 516-524. Print.

5. Corrado, Domenico, Cristina Basso, Maurizio Schiavon, and Gaetano Thiene. "Screening For Hypertrophic Cardiomyopathy In Young Athletes." New Eng- land Journal of Medicine 339.6 (1998): 364-369. Print

6. Woods, Lionel. Personal interview. 16 Jan. 2014

7. Mortazavi, Mohammed. "Sudden Cardiac Death in Young Athletes." Advances in Pediatrics 60.1 (2013): 201-215. Print.

8. Mues, C., L. Scherf, R. H. Strasser, and K. Ibrahim. "Survived Sudden Cardiac Death in a Young Marathon Runner: Cardiac Ischemic Event Attributed to an Interarterial Course of the Right Coronary Artery." Circulation 128.8 (2013): e104-e106. Print.

9. Wheeler MT, Heidenreich PA, Froelicher VF, Hlatky MA, Ashley EA. "Costeffectiveness of preparticipation screening for prevention of sudden cardiac death in young athletes." Ann Intern Med. 152(5):276-86. Print.

10. Azimi NA, Welch HG. "The effectiveness of cost-effectiveness analysis in containing costs." J Gen Intern Med. 1998;13(10):664-669. Print.

11. Charron P, Dubourg O, Desnos M, et al. "Diagnostic value of electrocardiography and echocardiography for familial hypertrophic cardiomyopathy in a genotyped adult population." Circulation. 1;96(1):214-9. Print.

12. Nagueh SF, Bierig SM, Budoff MJ, et al. "American Society of Echocardiography clinical recommendations for multimodality cardiovascular imaging of patients with hypertrophic cardiomyopathy: Endorsed by the American Society of Nuclear Cardiology, Society for Cardiovascular Magnetic Resonance, and Society of Cardiovascular Computed Tomography." J Am Soc Echocardiogr. 24;(5):473-98. Print.

13. Alter DA, Stukel TA, Newman A. "Proliferation of cardiac technology in Canada: a challenge to the sustainability of Medicare." Circulation. 24;113(3):380-7. Print.

14. Aatre, Rajini D., and Sharlene M. Day. "Psychological Issues in Genetic Testing for Inherited Cardiovascular Diseases." American Heart Association Journals 1 (2010): 3,8. Print.

15. LD, Cameron, and Muller C. "Psychosocial Aspects of Genetic Testing." National Centre for Biotechnology Information 1 (2009): 1. Print.

16. RD A, SM D. "Psychological issues in genetic testing for inherited cardiovascular diseases." Circ Cardiovasc Genet. 2011;4(1):81-90. Print.

17. Lampert R, Salberg L. Burg M. "Emotional Stress Triggers Symptoms in Hypertrophic Cardiomyopathy: A Survey of the Hypertrophic Cardiomyopathy Association." Pacing and Clinical Electrophysiology. 2010;33(9):1047-1053. Print.

18. Morse E, Funk M. "Preparticipation screening and prevention of sudden cardiac death in athletes: Implications for primary care." J Am Acad Nurse Pract. 2012;24(2):63-9. Print.

19. Pennington B. Hidden threats to young athletes. New York times. 2013 May 12, SP1.

20. Mortazavi M. "Sudden Cardiac Death in Young Athletes." Advances in Pediatrics. 2013;1(60):201-216. Print.

21. Maron BJ. "Hypertrophic Cardiomyopathy: a systematic review." JAMA. 13;287(10):1308-20. Print.

22. Cox S, O'Donoghue AC, McKenna WJ, et al. "Health related quality of life and psychological wellbeing in patients with hypertrophic cardiomyopathy." Heart. 78;2(2):182-7. Print.

23. Server ER, Sears SF, Nielson CD, et al. "Depression, anxiety and quality of life in patients with obstructive hypertrophic cardiomyopathy three months after alcohol septal ablation." Am J Cardiol. 15;100(10):1592-7. Print. 